\title{
Magnetic Nanoparticle-Based Hyperthermia for Head \& Neck Cancer in Mouse Models
}

\section{Qun Zhao ${ }^{1,2}{ }^{\varpi}$, Luning Wang ${ }^{1,2}$, Rui Cheng ${ }^{3}$, Leidong Mao ${ }^{3}$, Robert D. Arnold ${ }^{4}$, Elizabeth W. Howerth ${ }^{5}$, Zhuo G. Chen ${ }^{7}$, and Simon Platt 6}

1. Bioimaging Research Center, University of Georgia, Athens, GA. 30602, USA;

2. Department of Physics and Astronomy, University of Georgia, Athens, GA. 30602, USA;

3. Faculty of Engineering, Nano-scale Science and Engineering Center, University of Georgia, Athens, GA. 30602, USA;

4. Department of Pharmaceutical and Biomedical Sciences, University of Georgia, Athens, GA. 30602, USA;

5. Department of Pathology, University of Georgia, Athens, GA. 30602, USA;

6. Department of Small Animal Medicine \& Surgery, University of Georgia, Athens, GA. 30602, USA;

7. Winship Cancer Institute, Emory University, Atlanta, GA. 30322, USA

Corresponding author: Qun Zhao, PhD, Bioimaging Research Center and Department of Physics and Astronomy, University of Georgia, Athens, GA. 30602; qzhao@physast.uga.edu

(C) Ivyspring International Publisher. This is an open-access article distributed under the terms of the Creative Commons License (http://creativecommons.org/ licenses/by-nc-nd/3.0/). Reproduction is permitted for personal, noncommercial use, provided that the article is in whole, unmodified, and properly cited.

Received: 2011.11.22; Accepted: 2011.12.13; Published: 2012.01.15

\begin{abstract}
In this study, magnetic iron oxide nanoparticle induced hyperthermia is applied for treatment of head and neck cancer using a mouse xenograft model of human head and neck cancer (Tu2 2 cell line). A hyperthermia system for heating iron oxide nanoparticles was developed by using alternating magnetic fields. Both theoretical simulation and experimental studies were performed to verify the thermotherapy effect. Experimental results showed that the temperature of the tumor center has dramatically elevated from around the room temperature to about $40^{\circ} \mathrm{C}$ within the first 5-10 minutes. Pathological studies demonstrate epithelial tumor cell destruction associated with the hyperthermia treatment.
\end{abstract}

Key words: hyperthermia, magnetic nanoparticles, head and neck cancer, mouse

\section{Introduction}

The modern diagnosis and treatment of head and neck cancers (HNC) has now become more tailored to the individual patient and to specific cancer types than before. A single agent capable of diagnosing cancer, treating it and monitoring response to treatment simultaneously provides an ideal solution for the patients. In recent years, advances in tumor cells labeled with superparamagnetic iron oxide (SPIO) have provided increased understanding of the tumor biology and tissue microenvironment, while also offering great promise for the development of novel longitudinal monitoring and treatment evaluation methods of HNC patients [1-2].
Various strategies are used routinely in treatment of $\mathrm{HNC}$, including surgery, radiation therapy, and chemotherapy [3-4]. In recent years, ablative or thermotherapy has been developed for treatment of $\mathrm{HNC}$, offering patients an alternative and minimally invasive treatment option [5]. As a palliative treatment, the thermal treatment improves quality of life with decreased pain and preserves patient's function and appearance. In addition, there is a reduction in tumor bulk and analgesia requirements. Advantages include a reduction in procedural cost, avoidance of complex repetitive surgeries, and an ability to visualize the treated area at the time of the procedure. Ab- 
lation therapy is an evolving and exciting treatment option in the head and neck [6]. Among various methods of thermotherapy, magnetic fluid thermotherapy has drawn considerable attention [7-9]. In the magnetic fluid thermotherapy, magnetic fluids containing magnetic nanoparticles (e.g. iron oxide) are delivered to the cancer and then heated by external alternating magnetic field, resulting hyperthermia or thermo-ablation of cancer tissue. Recently, a magnetic alternating current hyperthermia $(\mathrm{MACH})$ device using external magnetic coils was reported for localized hyperthermia on head and neck cancer and lung cancer [10]. At the same time, magnetic nanoparticles based nanotherapy has been investigated for other types of tumors, for example, tumors of the central nervous system [11-12]. Additionally, nanotechnology has been applied extensively in drug delivery and therapy (including chemotherapy) [13-16], contrast-enhanced multifunctional imaging [17], tracking of stem cells [18], targeted molecular and biomolecular imaging [19-21], etc.

In this study, magnetic iron oxide nanoparticle ( $15 \mathrm{~nm}$ in diameter) induced hyperthermia is applied for treatment of head and neck cancer using a mouse xenograft model of human head and neck squamous cell carcinoma (HNSCC) cell line (Tu212). A hyperthermia system for remote heating of iron oxide nanoparticles was developed using alternating magnetic fields. Both theoretical simulation and experimental studies were performed to verify the thermotherapy effect. It is expected that this translational research will provide an alternative to the existing HNC treatment approaches.

\section{Methods and Materials:}

\section{Simulations of magnetic hyperthermia}

In the presence of time-varying magnetic field, magnetic nanoparticles will realign their magnetic moments to the applied field. The process of realignment is characterized by the relaxation time constants of nanoparticles, namely, Brownian and Néel time constants. As the excitation frequency of the magnetic field increases, magnetic moments of nanoparticles lag the applied field at a given angle. The resulting power dissipation process associated with this misalignment can increase bulk temperature of magnetic nanoparticles and their surroundings. This phenomenon can be used as a method of hyperthermia, suitable for cancer treatment in low-perfusion tissue [22-24].

Prior to experiments, theoretical simulation was carried out to estimate the temperature increase in a small low-perfusion tumor with volume of $1 \mathrm{~mL}\left(\mathrm{~V}_{\mathrm{t}}=\right.$
$1 \mathrm{~mL})$. Ferrofluid of volume $0.5 \mathrm{~mL}\left(\mathrm{~V}_{\mathrm{f}}=0.5 \mathrm{~mL}\right)$ were injected into the tumor and then heated up by high frequency alternating magnetic fields. We assumed the ferrofluid was localized as a spherical core at the center of the tumor by occupying porous tissue, therefore the total volume of the tumor after ferrofluid injection maintains at $1 \mathrm{~mL}$. This assumption was also adopted in previous models [17-18]. Furthermore, we assumed the ferrofluid was homogeneously distributed inside the spherical core with a radius of $4.9 \mathrm{~mm}$ $\left(\mathrm{R}_{\mathrm{f}}=4.9 \mathrm{~mm}\right)$. Additional simulation details are provided in the appendix.

\section{Cell and mice preparation}

Human HNSCC cell lines Tu212 [25] were cultured in DMEM/F12 (1:1) medium with 10\% (v/v) FBS in a humidified cell culture chamber (NuAire Inc. Plymouth, $\mathrm{MN}$ ) at $37^{\circ} \mathrm{C}, 5 \% \mathrm{CO}_{2}$. Cells were passaged when they reached approximately $80-90 \%$ confluency.

Five nude (NCr) mice (body weight, approx. 25 g) at 6-8 weeks of age were obtained from Taconic Farms, Inc., (Germantown, NY). Mice were maintained according to an approved Institutional Animal Care and Use Committee (IACUC) protocol at the University of Georgia and the U.S. Public Health Service (PHS) Policy on Humane Care and Use of Laboratory Animals, updated 1996. Mice were kept in pathogen-free cages in a light and temperature-controlled isolated room and provided with standard rodent chow and sterile water ad libitum during the experimental periods. Tu212 cells were harvested aseptically and a suspension $\left(2 \times 10^{7}\right.$ cells in 0.05 to $0.10 \mathrm{~mL}$ PBS) was injected subcutaneously into the flank of nude mice to establish tumor xenograft. Tumor growth and body weight were monitored every other day. Tumor volume was assessed using digital calipers as described previously where the volume was the product of largest dimension and (smallest dimension) ${ }^{2} \times 0.52$ [26]. After approximately 4 weeks the tumor volume reached approximately $1 \mathrm{~cm}^{3}$. Three mice were selected to receive the hyperthermia treatment as the experimental group, and the remaining two mice were kept as the control group, as described below. Mice were observed every other day until after tumor implantation and for $24-48 \mathrm{hr}$ after treatment and imaging for their general appearance as well as the treatment-mediated toxicities such as weight change, blood in stool, and decreased activity.

\section{Magnetic Resonance Imaging (MRI)}

MRI was performed to monitor the tumor before treatment. MR images were acquired using fast spin echo pulse sequences for the morphological studies of the tumors on a Varian 7 T 210mm bore MR scanner. 
Mice were anesthetized initially using 3\% isofluane mixed with oxygen in an induction chamber and maintained at $1.5 \%$ isofluane mixed with oxygen via a nose bane. The MRI parameters are: $\mathrm{TE}=10 \mathrm{~ms}, \mathrm{TR}=$ $2000 \mathrm{~ms}$, FOV $=40 \mathrm{~mm}$, slice thickness $=1 \mathrm{~mm}$, matrix size $=256 \times 256,7$ axial slices, gap $=1 \mathrm{~mm}$.

\section{Hyperthermia using an alternating magnetic field}

A solenoid coil $(4 \mathrm{~cm}$ in diameter and $10 \mathrm{~cm}$ in length) was wound on a plastic tube $(3.8 \mathrm{~cm}$ ID and $4.0 \mathrm{~cm}$ OD) using a gauge 16 copper wire. An impedance matching circuit, consisting of power resistors and capacitors, was made before the coil was driven by a power amplifier (Hafler Model\# P3000, AZ, USA). First, a signal generator produced a small sinusoidal signal of $130 \mathrm{kHz}$, which was then amplified to 9 A (peak to peak value) to generate an alternating magnetic field (AMF) around $7 \mathrm{kA} / \mathrm{m}$ inside the tube to heat SPIO nanoparticles. An optical fiber temperature probe (FISO, Quebec, Canada) was inserted to the tumor center during the treatment to monitor temperature.

After MRI, $0.5 \mathrm{~mL}$ ferrofluid of SPIO nanoparticles (magnetite $\left(\mathrm{Fe}_{3} \mathrm{O}_{4}\right)$ particles, with size of $\sim 15 \mathrm{~nm}$ in diameter and $5.8 \%$ volume fraction or an equivalent concentration of $0.30 \mathrm{~g} / \mathrm{mL}$, Ferrotec Co. EMG 705, $\mathrm{NH}, \mathrm{USA}$ ) was used as hyperthermia media. The fluid was delivered through intratumoral injection for the experimental group (6 mice). Figure 1 illustrates the hyperthermia experimental scheme, where the mouse is placed inside the solenoid coil (blue color) with tumor positioned at the coil center in order to expose the tumor to the strongest magnetic field generated by the coil. The control group ( 2 mice) went through the hyperthermia but without nanoparticles injected.

\section{Estimate of SAR in hyperthermia experiment}

The SAR (Specific Absorption Rate) is used to describe the quantification of heat generation from magnetic particles. Experimentally, it can be measured by the following equation [1]:

$$
S A R=C\left(\frac{\Delta T}{\Delta t}\right)_{i} \frac{m_{f}}{m_{p}}
$$

Where $\mathrm{C}=1500 \mathrm{~J} /(\mathrm{kg} . \mathrm{K})$ is mass based specific heating capacity of mixture of ferrofluid and tissue fluids [27], $\Delta \mathrm{T}$ is the amount of temperature change over time $\Delta t$, the ratio of $(\Delta \mathrm{T} / \Delta \mathrm{t})_{\mathrm{i}}$ is the initial gradient or slope of a heating curve (temperature vs. time), $\mathrm{m}_{\mathrm{f}}=1.19 \mathrm{~g} / \mathrm{mL}$ is the density of ferrofluid, and $m_{p}=0.3 \mathrm{~g} / \mathrm{mL}$ is the density of $\mathrm{Fe}_{3} \mathrm{O}_{4}$ in the fluid.

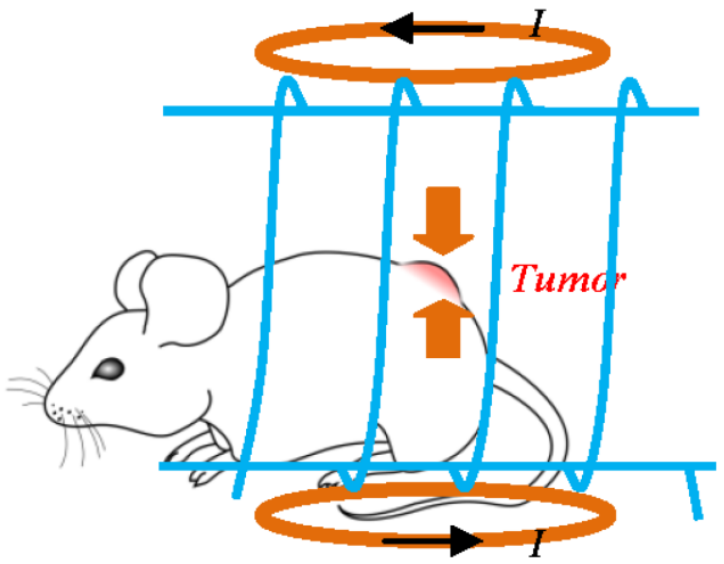

Figure I Illustration of the hyperthermia experimental scheme, where the mouse is placed inside the solenoid coil (blue color) with tumor positioned at the coil center in order to expose the tumor to the strongest magnetic field generated by the coil.

\section{Preparation of tumor tissues}

At 24-48 hours post treatment mice were sacrificed and tumor tissues were removed. Removed tumor tissues were fixed in $10 \%$ formalin and routinely processed and embedded in paraffin. Sections were cut and stained with hematoxylin and eosin (HE) and Perl's iron stain. For the Perl's iron stain, 4-5 micron sections were microwaved in potassium ferrocyanide hydrochloric acid solution for 30 seconds, rinsed in deionized water, and counterstained with nuclear fast red Kernechtrot solution for 3-5 min, rinsed in tap water, dehydrated and mounted. Additional sections were stained by caspase 3 (cleaved) immunohistochemistry to detect apoptosis. Briefly, 4-5 micron sections were deparaffinized, heated at 120 degrees for 8 minutes in Tris/EDTA High pH 9.0 buffer, incubated with rabbit anti-Caspase 3 (cleaved) antibody (1:200 dilution for $60 \mathrm{~min}$;Biocare, Concord, CA), and blocked with power block (Biogenex, San Ramon, CA). Antibody was then detected with an streptavidin-biotin alkaline phosphatase method (biotinylated anti-rabbit;Vector Labs, Burlingame, CA and alkaline phosphatase labeled streptavidin; Biogenex, San Ramon, CA) and Warp Red chromogen (Biocare, Concord, CA).

\section{Results}

\section{Simulation and experimental results of hy- perthermia}

Figure 2 presents the simulation model and results. Simulation parameters are chosen to match the experimental conditions. Fig. 2(a) shows the location 
of ferrofluids core (inner circle, $\mathrm{V}_{\mathrm{f}}=0.5 \mathrm{~mL}$ ) within a spherical tumor (outer circle, $\mathrm{V}_{\mathrm{t}}=1 \mathrm{~mL}$ ). Magnetic field frequency is $130 \mathrm{kHz}(\mathrm{f}=130 \mathrm{kHz})$, and field strength is $7000 \mathrm{~A} / \mathrm{m}$ (peak to peak value $\mathrm{H}=7000$ A/m). Fig. 2(b) depicts the temperature increase over time at the center of the tumor, where the thermal probe is located in the subsequent experiments. Based on our experimental conditions, the temperature of the tumor is predicted to increase $\sim 20$ degrees with 10 minutes application of magnetic fields, which matches the experimental results reasonably well, as detailed below.
Figure 3 displays the in vivo experimental results. Fig. 3(a) shows MR image of one of tumors (circled and labeled) in the experiment group before treatment. Fig. 3(b) presents the temperature curves of experimental group tumors (4 out of the total of 6 ) during the hyperthermia treatment. Based on the graph, the temperature of the tumor center has dramatically elevated from around the room temperature to about $40^{\circ} \mathrm{C}$ within the first $5-10$ minutes. Then, the amount of current flowing in the solenoid coil was controlled to maintain the regional temperature between $40^{\circ} \mathrm{C}$ to $50^{\circ} \mathrm{C}$. The entire hyperthermia treatment took approximately 20 minutes.

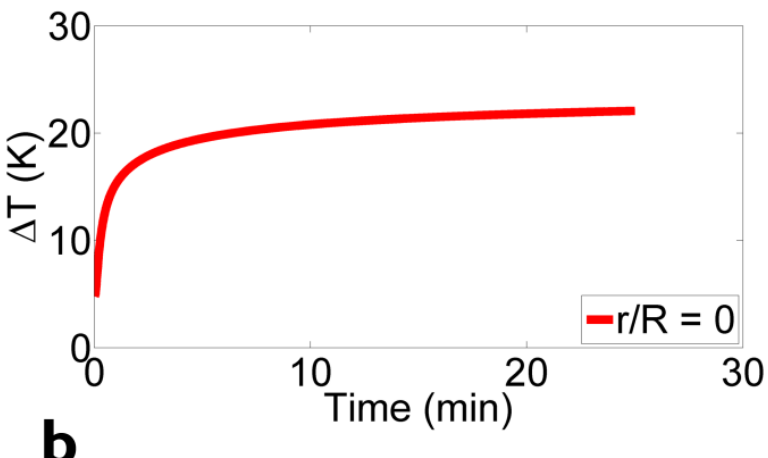

Figure 2 presents the simulation model and results. Simulation parameters are chosen to match the experimental conditions. (a) shows the location of ferrofluids core (inner circle, $\mathrm{V}_{\mathrm{f}}=0.5 \mathrm{~mL}$ ) within a spherical tumor (outer circle, $\mathrm{V}_{\mathrm{t}}=1 \mathrm{~mL}$ ). Magnetic field frequency is $130 \mathrm{kHz}(\mathrm{f}=130 \mathrm{kHz}$ ), and field strength is $7000 \mathrm{~A} / \mathrm{m}$ (peak to peak value $\mathrm{H}=7000 \mathrm{~A} / \mathrm{m}$ ). (b) depicts the temperature increase over time at the center of the tumor, where the thermal probe is located in the subsequent experiments.
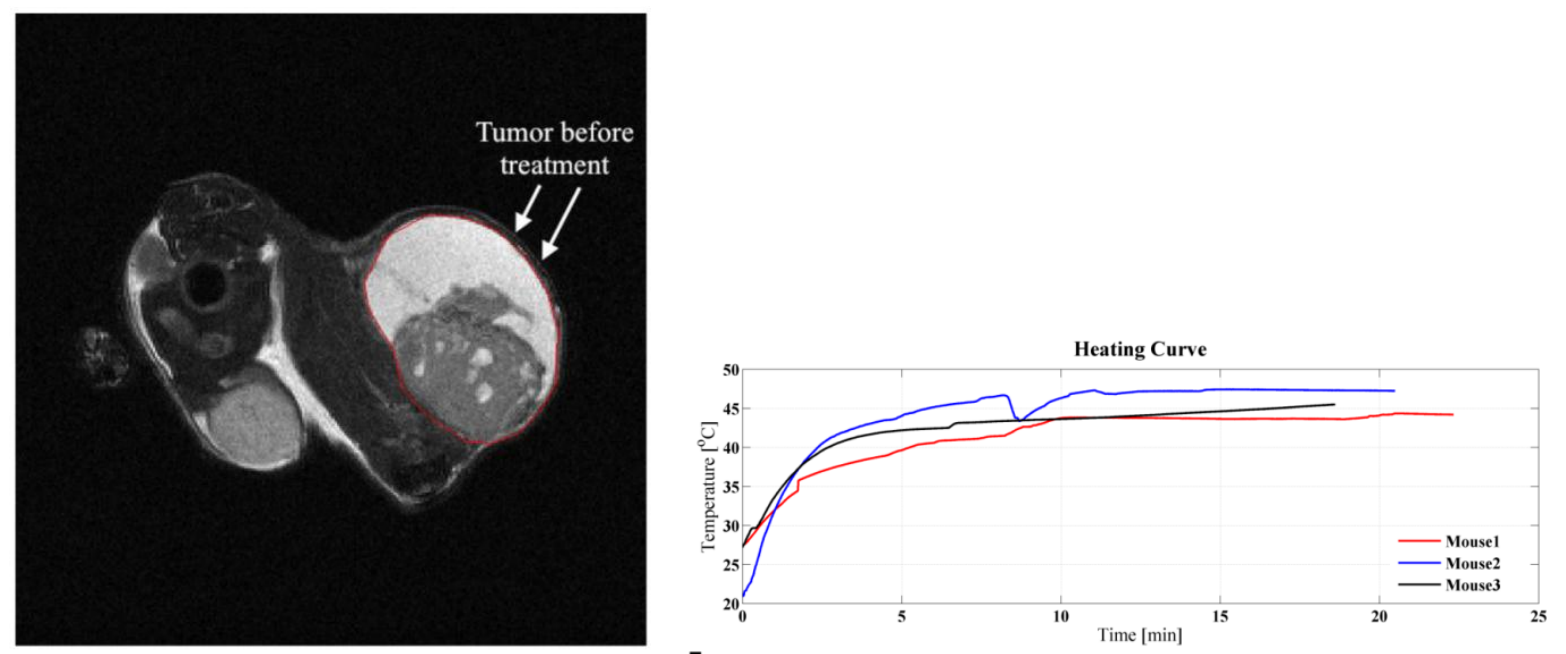

a

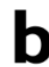

Figure 3 In vivo experimental results: (a) shows the mouse MR image (tumor labeled) in the experiment group before treatment. (b) presents the temperature curves of experimental group tumors during the hyperthermia treatment. Based on the graph, the temperature of the tumor center has dramatically elevated from around the room temperature to about $40^{\circ} \mathrm{C}$ within the first 5 minutes, then temperature maintains in between $40^{\circ} \mathrm{C}$ to $50^{\circ} \mathrm{C}$. The entire hyperthermia treatment took approximately 20 minutes. 
Table 1 gives the SAR estimate (eq. 1) based on the experimental temperature curve of Fig. 3(b). The column 2-4 list the temperature measurement at 0.5 $\min \left(T_{1}\right), 2.5 \min \left(T_{2}\right)$, temperature gradient or slope, and SAR estimate. As a comparison to the in vivo results, 3 pure ferrofluid samples were made of EMG $705(0.5 \mathrm{~mL})$ sealed in a $1 \mathrm{~mL}$ plastic vial. They were heated with the designed coil using the same frequency and magnetic field strength as those of the mouse experiments. The column 5-7 list the corresponding results.

\section{Histopathology}

Both treated and nontreated tumors were composed of one or more circumscribed nodules that had a peripheral wall of neoplastic stratified squamous epithelium, which differentiated centripetally, and central necrosis. Treated tumors had variable degrees of ulceration and inflammation of the tumor wall as compared to the nontreated control tumors that had none (Figure 4). Remaining epithelium in the wall of treated tumors was mostly viable, but coagulative necrosis was present in one. Treated tumors also had more severe inflammation around the tumor and within the wall and centers of the tumors than the nontreated controls. No stainable iron was observed in the nontreated controls. However, the treated tumors had abundant iron which was free and intracellular (cytoplasmic and nuclear) in the tumor centers, rarely in the cytoplasm and nuclei of the wall, and then only in necrotic cells, and around the tumors as extracellular lakes and cytoplasmic in macrophages, fibroblasts and adipocytes (Figure 5). Caspase 3 staining was similar between the treated and nontreated tumors, with staining of multiple small groups of epithelial cells and inflammatory cells at the interface between the wall epithelium and in the central area of necrosis (Figure 6).

Table I. Estimation of SAR based on the in vivo experiments and pure ferrofluid samples. The column 2-4 list the in vivo experiments, and column 5-7 list those of pure ferrofluid samples. Row 2-6 present the temperature measurements at 0.5 $\min \left(T_{1}\right), 2.5 \min \left(T_{2}\right)$, temperature gradient or slope, SAR estimate, and average SAR.

\begin{tabular}{|c|c|c|c|c|c|c|}
\hline & Mouse 1 & Mouse 2 & Mouse 3 & Vial 1 & Vial 2 & Vial 3 \\
\hline $\mathrm{T}_{1}\left({ }^{\circ} \mathrm{C}\right)$ & 26.0 & 29.5 & 28.2 & 27.7 & 30.8 & 28.4 \\
\hline $\mathrm{T}_{2}\left({ }^{\circ} \mathrm{C}\right)$ & 40.5 & 39.0 & 36.5 & 57.8 & 60.8 & 58.2 \\
\hline $\begin{array}{c}(\Delta \mathrm{T} / \Delta \mathrm{t})_{\mathrm{avg}} \\
\left({ }^{\circ} \mathrm{C} / \mathrm{min}\right)\end{array}$ & 7.3 & 4.7 & 4.2 & 15.1 & 15.0 & 14.9 \\
\hline SAR(W/kg) & 723.9 & 466.1 & 416.5 & 1497.5 & 1487.6 & 1477.6 \\
\hline $\begin{array}{l}\text { Average SAR } \\
(\mathrm{W} / \mathrm{kg})\end{array}$ & 535.5 & & & 1487.6 & & \\
\hline
\end{tabular}
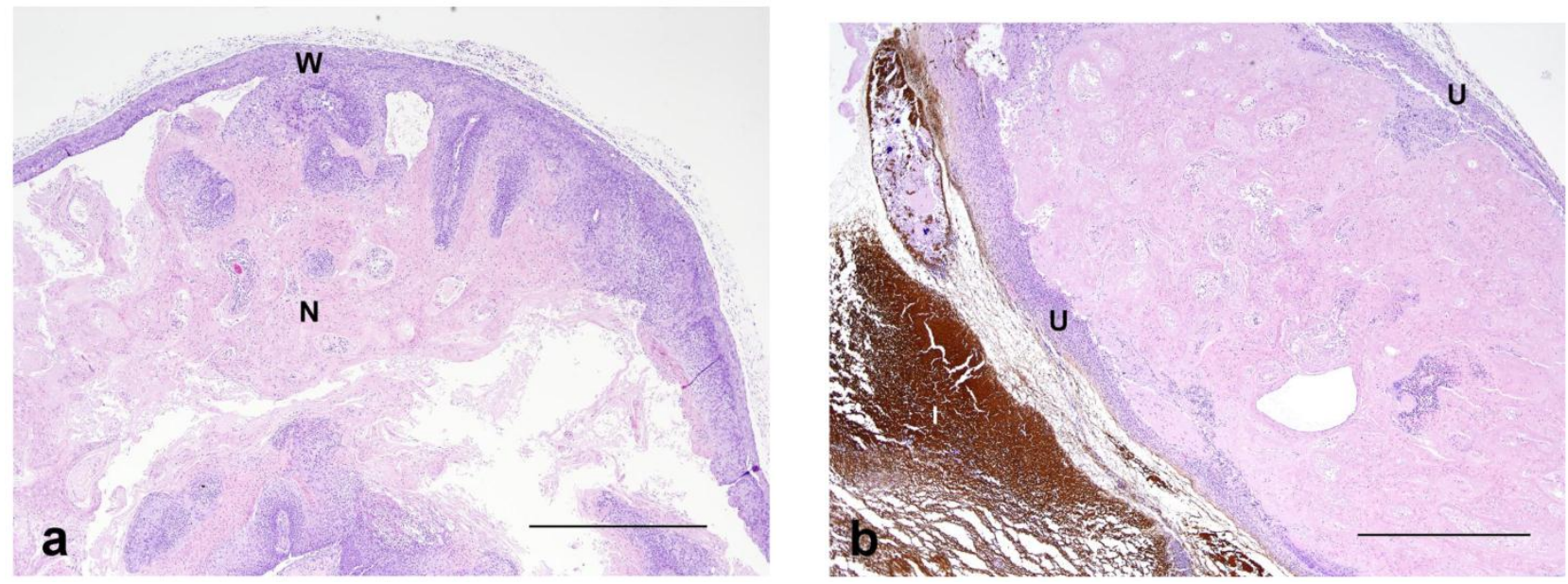

Figure 4 (a): Nontreated tumor without ulceration of the tumor wall. Note intact epithelium forming wall (W) and necrotic center $(\mathrm{N})$ compared to (b): Treated tumor with severe ulceration of the wall with extensive loss of epithelium, indicative of cellular destruction, and infiltration with inflammatory cells (U). Note brown peritumoral iron (I) at the lower-left (in white color). HE stain. Bar= $1 \mathrm{~mm}$. 

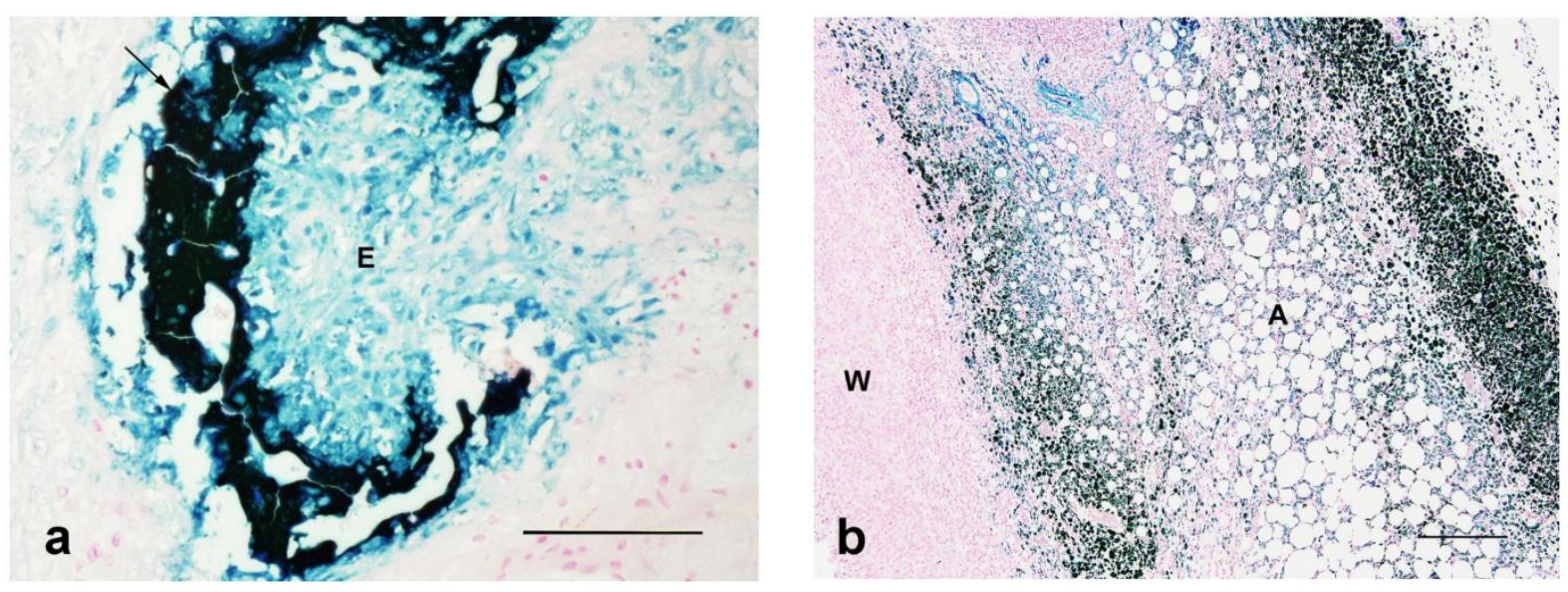

Figure 5 (a): Center of treated tumor demonstrating cytoplasmic and nuclear iron staining (seen as blue or green) in necrotic epithelium (E) and extracellular iron (arrow). Perl's iron stain. Bar= 100 microns; (b): Extensive iron staining (seen as blue or green) around a tumor that is cytoplasmic in macrophages, fibroblasts, and adipose tissue (A). Tumor wall (W) does not have any iron staining. Perl's iron stain. Bar $=200$ microns.

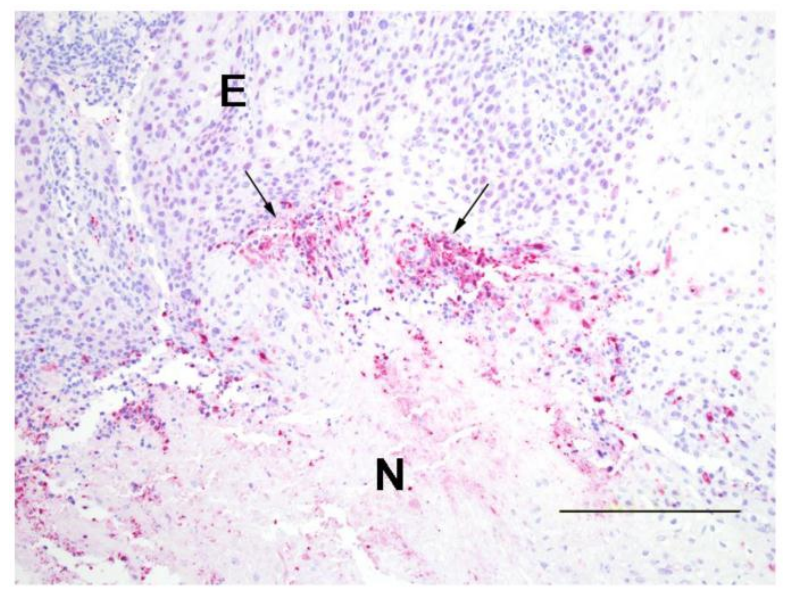

Figure 6 Caspase 3 immunohistochemical staining was similar between treated and nontreated tumors occurring at the interface (arrows) of the epithelium $(E)$ of the wall and the necrotic center $(N)$. Shown is a treated tumor. Avidin-biotin alkaline phosphatase technique. Warp red chromogen and hematoxylin counterstain. Bar=200 microns.

\section{Discussion and Conclusion}

In this study we demonstrated that magnetic heating of iron oxide nanoparticles can be achieved by applying an alternating magnetic field. This was applied to a mouse xenograft model of human HNSCC (Tu212 cell line).

The designed solenoid coil generates the strongest and most uniform magnetic field around the center of the coil. The thermal power density is proportional to the square of magnetic field $\mathrm{H}$ (eq. (1) of the Appendix). It is anticipated that the optimal hyperthermia is generated when the tumor is positioned at the center of the coil. As seen in the Table 1, the SAR of in vivo experiments $(535 \mathrm{~W} / \mathrm{kg})$ is about one third of that $(1487 \mathrm{~W} / \mathrm{kg})$ generated by pure ferrofluid samples. The lower SAR of in vivo result is possibly due to heat transfer between the tumor and surrounding tissues, and fast heat dissipation because of the blood flow supplied to the tumor. Similar studies reported SAR of $10-80 \mathrm{~W} / \mathrm{g}$ depending on magnetite $\left(\mathrm{Fe}_{3} \mathrm{O}_{4}\right)$ particle size and alternating magnetic field frequency. [28]

To improve heating efficiency and making sure the heating is focused on tumor only, a second coil can be applied to generate a static field magnetic field by using a pair of Maxwell coils (shown in orange color as in the Fig.1). A counter-rotating direct current (dc) running in the Maxwell pair of coils generates a roughly linear magnetic field with zero-crossing (i.e., zero magnetic field) at the middle of the two coils. When the tumor to be treated is placed around the 
middle of the Maxwell coil, the alternating magnetic field (generated by the solenoid coil in blue color as shown in Fig.1) functions as designed to heat the nanoparticles populated in the tumor. However, magnetic particles diffused or carried away from the tumor by blood flow are not heated. This is because when the particles are positioned in the static magnetic field generated by the Maxwell coil, the static field (with equal or larger amplitude) is superimposed on the alternating magnetic field, Neel and Brownian relaxations of the particles are blocked and heating will be diminished. This will significantly reduce heating to the normal tissues surrounding the tumors.

In this proof of concept study, magnetic nanoparticles were delivered through intratumoral injection in order to keep the concentration desired for hyperthermia effect. A systemic delivery of the nanoparticles to the tumor by specific antibodies was reported by other studies. [9]

As seen in the histopathology results, severe ulceration of the wall of treated tumors which was not seen in the nontreated controls can be attributed to cell death caused by the hyperthermia. The presence of coagulative necrosis of the epithelium in the wall of one treated tumor and no difference in caspase staining between treated and nontreated tumors indicates that most hyperthermia-mediated cell death is due to oncotic necrosis and not apoptosis. Severe inflammation in the treated tumors can also be explained by the hyperthermia-associated necrosis.

The thermal therapy can be combined with chemotherapy and radiation for a potentially better treatment effect for HNC patients. In addition to as hyperthermia media, the magnetic nanoparticles can also function as drug carriers for an image-guided delivery. [15]

In summary, we demonstrated in this study that hyperthermia using magnetic heating of iron oxide nanoparticles can be achieved by applying an alternating magnetic field. In vivo experiments using mouse xenograft models showed that the temperature of tumor center was elevated quickly to cause hyperthermia-mediated cell death due to oncotic necrosis.

\section{Supplementary Material}

Appendix: Mathematical Treatment of the simulation of heating experiment.

http://www.thno.org/v02p0113s1.pdf

\section{Acknowledgement}

This study was supported by the Head \& Neck SPORE development research program, Winship
Cancer Institute, the Emory University (PI Zhao \& Platt).

\section{Conflict of Interest}

The authors have declared that no conflict of interest exists.

\section{References}

1. Liu W, Frank JA. Detection and quantification of magnetically labeled cells by cellular MRI. Eur J Radiol. 2008; 70: 258-64.

2. Dixon WT, Blezek DJ, Lowery LA, Meyer DE, Kulkarni AM, Bales BC, et al. Estimating amounts of iron oxide from gradient echo images. Magn Reson Med. 2009; 61: 1132-6.

3. Haddad RI, Shin DM. Recent advances in head and neck cancer. N Engl J Med. 2008; 359: 1143-54.

4. Shin DM, Khuri FR. Advances in the management of recurrent or metastatic squamous cell carcinoma of the head and neck. Head Neck. 2011; epub.

5. Ang KK, Andratschke NH, Milas L. Epidermal growth factor receptor and response of head-and-neck carcinoma to therapy. Int J Radiat Oncol Biol Phys. 2004; 58: 959-65.

6. Wyse G, Hong H, Murphy K. Percutaneous thermal ablation in the head and neck: current role and future applications. Neuroimaging Clin N Am. 2009; 19: 161-8.

7. Moroz P, Jones SK, Gray BN. Magnetically mediated hyperthermia: current status and future directions. Int J Hyperthermia. 2002; 18: 267-84.

8. Tasci TO, Vargel I, Arat A, Guzel E, Korkusuz P, Atalar E. Focused RF hyperthermia using magnetic fluids. Med Phys. 2009; 36: 1906-12.

9. Ivkov R, DeNardo SJ, Daum W, Foreman AR, Goldstein RC, Nemkov VS, et al. Application of high amplitude alternating magnetic fields for heat induction of nanoparticles localized in cancer. Clin Cancer Res. 2005; 11: 7093s-103s.

10. Thomas LA, Dekker L, Kallumadil M, Southern P, Wilson M, Nair SP, et al. Carboxylic acid-stabilised iron oxide nanoparticles for use in magnetic hyperthermia. J Mater Chem. 2009; 19: 6529-35.

11. Muller S. Magnetic fluid hyperthermia therapy for malignant brain tumors--an ethical discussion. Nanomedicine. 2009; 5: 387-93.

12. Provenzale JM, Silva GA. Uses of nanoparticles for central nervous system imaging and therapy. AJNR Am J Neuroradiol. 2009; 30: 1293-301.

13. Wang X, Yang L, Chen ZG, Shin DM. Application of nanotechnology in cancer therapy and imaging. CA Cancer J Clin. 2008; 58: 97-110.

14. Cho K, Wang X, Nie S, Chen ZG, Shin DM. Therapeutic nanoparticles for drug delivery in cancer. Clin Cancer Res. 2008; 14: 1310-6.

15. Lammers T, Kiessling F, Hennink WE, Storm G. Nanotheranostics and image-guided drug delivery: current concepts and future directions. Mol Pharm. 2010; 7: 1899-912.

16. Mahmoudi M, Sant S, Wang B, Laurent S, Sen T. Superparamagnetic iron oxide nanoparticles (SPIONs): development, surface modification and applications in chemotherapy. Adv Drug Deliv Rev. 2011; 63: 24-46.

17. Jin $Y$, Jia C, Huang SW, O'Donnell M, Gao X. Multifunctional nanoparticles as coupled contrast agents. Nat Commun. 2010; 1: 41.

18. Mahmoudi M, Hosseinkhani H, Hosseinkhani M, Boutry $S$, Simchi A, Journeay WS, et al. Magnetic resonance imaging tracking of stem cells in vivo using iron oxide nanoparticles as a tool for the advancement of clinical regenerative medicine. Chem Rev. 2011; 111: 253-80. 
19. Lee S, Xie J, Chen X. Peptide-based probes for targeted molecular imaging. Biochemistry. 2010; 49: 1364-76.

20. Mahmoudi M, Serpooshan V, Laurent S. Engineered nanoparticles for biomolecular imaging. Nanoscale. 2011; 3: 3007-26.

21. Mather S. Molecular imaging with bioconjugates in mouse models of cancer. Bioconjug Chem. 2009; 20: 631-43.

22. Rosensweig RE. Heating magnetic fluid with alternating magnetic field. J Magn Magn Mater 2002; 252: 370-4.

23. Cantillon-Murphy P, Wald LL, Zahn M, Adalsteinsson E. Proposing Magnetic Nanoparticle Hyperthermia in Low-Field MRI. Concept Magn Reson A. 2010; 36A: 36-47.

24. Cantillon-Murphy P, Wald LL, Adalsteinsson E, Zahn M. Heating in the MRI environment due to superparamagnetic fluid suspensions in a rotating magnetic field. J Magn Magn Mater. 2010; 322: 727-33.

25. Chen Z, Zhang X, Li M, Wang Z, Wieand HS, Grandis JR, et al. Simultaneously targeting epidermal growth factor receptor tyrosine kinase and cyclooxygenase-2, an efficient approach to inhibition of squamous cell carcinoma of the head and neck. Clin Cancer Res. 2004; 10: 5930-9.

26. Davol PA, Frackelton AR, Jr. Targeting human prostatic carcinoma through basic fibroblast growth factor receptors in an animal model: characterizing and circumventing mechanisms of tumor resistance. Prostate. 1999; 40: 178-91.

27. Sawyer CA, Habib AH, Miller K, Collier KN, Ondeck CL, McHenry ME. Modeling of temperature profile during magnetic thermotherapy for cancer treatment. J Appl Phys. 2009; 105: 07B320

28. Purushotham S, Ramanujan RV. Modeling the performance of magnetic nanoparticles in multimodal cancer therapy. J Appl Phys. 2010; 107: 114701-9.

29. Andra W, d'Ambly CG, Hergt R, Hilger I, Kaiser WA. Temperature distribution as function of time around a small spherical heat source of local magnetic hyperthermia. J Magn Magn Mater. 1999; 194: 197-203.

\section{Author Biography}

Qun Zhao, PhD, is an assistant Professor of Physics at the Bioimaging Research Center, University of Georgia. He received his $\mathrm{PhD}$ in electrical engineering from Xidian University in 1995. Then he performed postdoctoral work in magnetic resonance physics at the University of Florida during 1998-2001. He joined the University of Georgia in 2006 before worked in the MR industry during 2001-05. He has been working over ten years on magnetic resonance imaging (MRI), contrast-enhanced MRI, quantitative MRI of cancer using magnetic nanoparticles, and signal/image processing. His research has been supported by the National Institutes of Health, Department of Defense, and UGA research foundation. He has over thirty publications in peer-reviewed journals and over fifty conference proceeding and abstracts.

Luning Wang is a doctoral student in the Department of Physics and Astronomy. He obtained his B.S. degree in 2008 from Zhengzhou University, China. His research interest is magnetic resonance physics including pulse sequences and quantification of tissue susceptibility. He currently works with Dr. Qun Zhao in the MRI physics lab.
Rui Cheng is a PhD student in Faculty of Engineering at the University of Georgia. He obtained his B.S. degree from Tsinghua University in China, and his M.S. degree from the University of Minnesota. He currently works in Dr. Leidong Mao's laboratory.

Leidong Mao, PhD, is an Assistant Professor of Faculty of Engineering at the University of Georgia. He received his B.S. degree at Fudan University in China. He received M.S., M.Phil., and Ph.D. degrees in electrical engineering from Yale University in 2002, 2005 and 2007. He has over 20 publications on ferrofluid synthesis, modeling, and applications. His research has been supported by CDC and UGA research foundation.

Robert D. Arnold, $\mathrm{PhD}$, is an Assistant Professor of Pharmaceutical and Biomedical Sciences at the University of Georgia (UGA), College of Pharmacy. He obtained a Ph.D (2004) in Pharmaceutics at the University at Buffalo (UB). A broad goal of his research is to optimize chemotherapeutic dosing schedules and exploit differences in tumor microenvironment to target disposition and modulate drug release kinetics of multifunctional nanoparticulate drug-carriers. The goal is to integrate the pathophysiology of a disease state with development of drug-carriers to maximize a drugs pharmacological activity and minimize off-target toxicity in the treatment of primary and metastatic cancers. Dr. Arnold was inducted into the Rho Chi Pharmacy Honor Society (2009) and was named a Georgia Cancer Coalition Distinguished Research Cancer Scholar (2007). His research has been supported the American Foundation for Pharmaceutical Education, the National Institute of Biomedical Imaging and Bioengineering, and a variety of local and private institutions.

Elizabeth W. Howerth. B.S. from University of Florida (1975), DVM from Michigan State University (1978), and PhD from University of Georgia (1986). Diplomate American College of Veterinary Pathologists. Current position: Professor, Department of Pathology, College of Veterinary Medicine, University of Georgia, Athens, GA. Research interests include pathobiology of infectious diseases of large animals and wildlife, veterinary neurologic diseases, and cancer in animals.

Zhuo G. Chen received her PhD from Louisiana State University, USA, in 1989. Dr. Chen's research focuses on the metastasis of head and neck cancer, signal transduction pathways in cancer cells prognostic and therapeutic biomarkers, anticancer therapeutic drugs for treatment of cancer and usage of nanotechnology in cancer research and clinical application. As Principal Investigator (PI) in the study of head and neck cancer, her work has been supported over the 
past few years by several awards from the US National Institutes of Health (NIH). Currently, she is a Project Leader for the Head and Neck Cancer SPORE supported by NIH, and a project funded by the US Department of Defense. She also served as PI on a project for identifying metastasis-related proteins in head and neck cancer using nanotechnology, and as a co-investigator for several projects related to prevention and treatment of lung and head and neck cancers using targeted small molecules and nanotherapeutic drugs. These studies have generated 83 publications in peer-reviewed journals.

Simon Platt is an Associate Professor of Neurology at the University of Georgia. He qualified from the University of Edinburgh in 1992 at which time he undertook a one year internship in small animal medicine and surgery at the University of Guelph in Canada. After spending two years in small animal practice in the UK, Simon began a three year residency in neurology and neurosurgery at the University of Florida. In 1998, he was appointed assistant professor of Neurology / Neurosurgery at the University of Georgia. In 2000, he became head of the neurology unit at the Animal Health Trust in Newmarket where he stayed until 2007 at which time he moved back to UGA as associate professor of neurology/neurosurgery. Simon is a Diplomate of the American College of Veterinary Internal Medicine in Neurology and of the European College of Veterinary Neurology (ECVN) as well as being a RCVS recognized specialist in Veterinary Neurology. Simon is currently the ACVIM Neurology Specialty President. $\mathrm{He}$ is also co-editor of the BSAVA manual of Canine and Feline Neurology and the forthcoming Manual of Small Animal Neurological Emergencies. 